J Neurol Neurosurg Psychiatry 2003;74(Suppl I):i42-i44

M anagement of children with high flow arteriovenous shunts of the brain is among the most challenging areas in modern medicine. Intracranial arteriovenous shunts (AVS) in children differ considerably from those seen in adults, in whom brain arteriovenous malformations (AVMs) and acquired dural arteriovenous fistulae predominate. These differences are seen both in the types of lesion and in their effects. In the neonatal and infantile age groups, the most common type of AVS is the vein of Galen aneurysmal malformation (VGAM), which has a male-to-female ratio of 3:1. Progressing further into childhood, dural malformations and brain arteriovenous malformations become more common. ${ }^{1}$ The consequences of an AVS in the developing brain are different from those in an adult, principally because of the immature cerebral venous system: the arachnoid granulations by which cerebrospinal fluid will be returned to the cerebral venous sinuses are not fully matured until 16-18 months of age. ${ }^{2}$ In infancy, cerebrospinal fluid is reabsorbed across the ventricular ependyma and brain parenchyma into the medullary veins. The presence of a large AVS such as a VGAM may raise venous sinus pressure, which is transmitted in turn to the cortical and finally the medullary veins. This will result in water congestion of the brain parenchyma, and impaired oxygenation leading to subependymal atrophy and in severe cases a progressive "melting brain syndrome". ${ }^{3}$ The most common presentation of VGAM results from the size of the shunt itself, imposing elevated preload on the right side of the heart leading to cardiac failure. This may progress to multisystem failure. Haemorrhage in children with VGAMs is rare. ${ }^{4}$

These are rare lesions and experience in their management has been restricted generally to large paediatric centres where a close collaboration between neuroradiologists, neonatologists, paediatric cardiologists, and neurologists has been achieved. Foremost among these centres has been Bicetre Hospital in Paris where Professor Pierre Lasjaunias' group has done much to clarify the nature of the disease and its appropriate management. A typical neurosciences unit serving a population of about three million could expect approximately one new VGAM patient a year. The aetiology of VGAMs is unknown; however, an early insult, perhaps resulting in a somatic mutation in neural crest and/or adjacent cephalic mesoderm in the early embryo, could be expected to cause such vascular abnormalities. ${ }^{5}$

\section{ANATOMY OF VGAM}

The VGAM is a rare and dramatic form of embryonic arteriovenous shunt located in the midline in the choroidal fissure. It consists of multiple feeding arteries, principally the anterior and posterior choroidal arteries and the anterior cerebral artery (following the cingulate gyrus and representing the embryonic limbic arterial arch), draining directly into an enlarged venous pouch (fig 1 ). This usually grossly dilated vein was recognised by Raybaud and colleagues to be the embryonic precursor of the vein of Galen-the median vein of the prosencephalon. ${ }^{6}$ This malformation develops before the formation of the vein of Galen and straight sinus, and the pouch drains via a falcine sinus to the superior sagittal sinus. The deep venous system of the brain does not usually communicate with the venous pouch, but rather drains by mesencephalic collateral veins often visible with an epsilon shape (fig 2). The VGAM should be distinguished from aneurysmal dilatation of the true vein of Galen caused by an adjacent brain AVM (vein of Galen aneurysmal dilatation or VGAD) which has a higher risk of haemorrhage.

See end of article for authors' affiliations

Correspondence to Dr JJ Bhattacharya, Department of Neuroradiology, Institute of Neurological Sciences, Southern General Hospital, Glasgow G51 4TF, UK. gc1232@clinmed.gla.ac.uk
VGAMs are occasionally detected on antenatal ultrasound scans (from about 25 weeks gestation) as apparently cystic midline brain lesions, colour flow Doppler then suggesting a VGAM. Antenatal magnetic resonance imaging will confirm the diagnosis and allow assessment of any pre-existing damage to the brain (fig 3). It will also allow treatment planning with delivery at a centre with the appropriate facilities and expertise, principally fetal medicine, neonatology, paediatric cardiology and intensive care, and interventional neuroradiology. A team approach is critical to successful management.

\section{PRESENTATION AND DIAGNOSIS OF VGAMS}




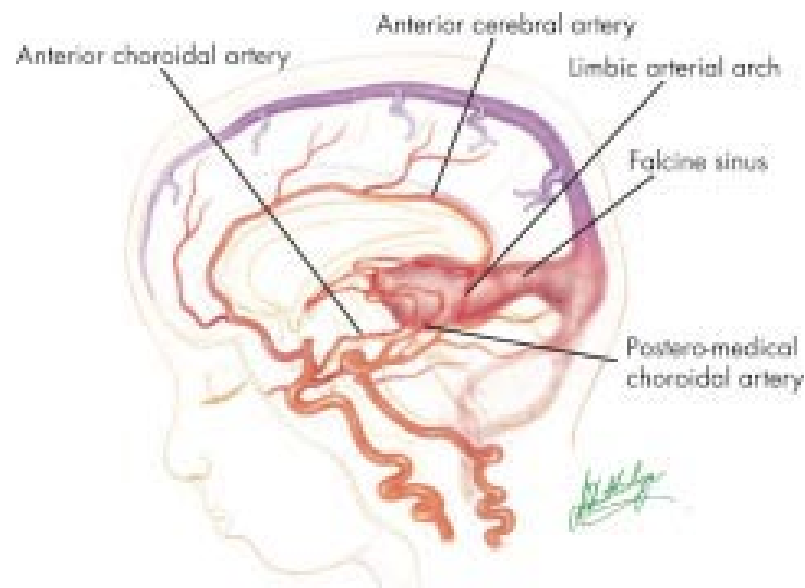

Figure 1 Schematic illustration of the most common choroidal type of vein of Galen aneurysmal malformation (VGAM). The shunt has multiple feeding arteries including the anterior cerebral artery, and anterior and posterior choroidal arteries. The enlarged midline venous sac at the normal location of the vein of Galen represents a dilated median vein of the prosencephelon, draining via an enlarged falcine sinus. The normal straight sinus has not formed.

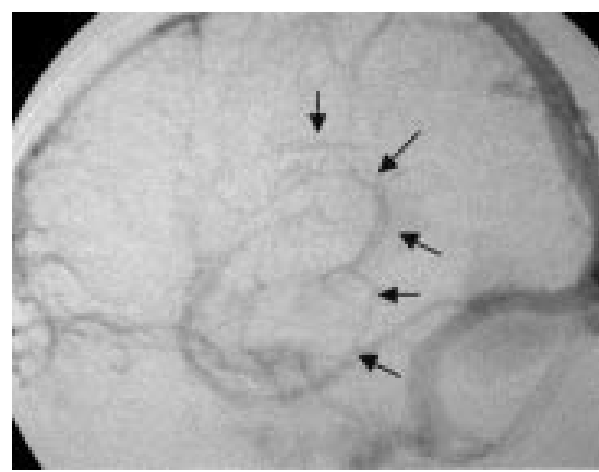

Figure 2 Venous phase of an angiogram in an infant with VGAM The internal cerebral veins do not communicate with the enlarged venous pouch (medial vein of the prosencephalon) but drain inferiorly into the mesencephalic anastomotic veins (arrows) giving a characteristic "epsilon" or figure 3 configuration.

More commonly VGAMs are diagnosed after birth. Often delivery and the first 24 hours are unremarkable. Larger shunts may then show rapid deterioration with progressively worsening cardiac failure leading on to multiorgan failure. A smaller shunt may present later with mild cardiac failure and failure to thrive. Commonly, initial investigations seek a cardiac cause for the high output failure. When cardiac examination is normal, transfontanelle ultrasound will detect the VGAM. Occasional children present later in childhood with macrocrania or prominent facial veins secondary to venous outflow obstruction. Initial assessment of the AV shunt should be by magnetic resonance imaging or computed tomography. Angiography is best performed at the time of embolisation.

\section{TREATMENT AND OUTCOME IN VGAMS}

Ideally the initial treatment of VGAM is conservative. Embolisation of a neonate is a high risk procedure and where possible we treat the child medically (for cardiac failure) until aged 5 or 6 months with regular outpatient assessment. Elective embolisation can be scheduled for this time with the aim of closing the AVS with cyanoacrylate glue by the arterial route. If the infant deteriorates (seizures, failure to thrive, worsening cardiac

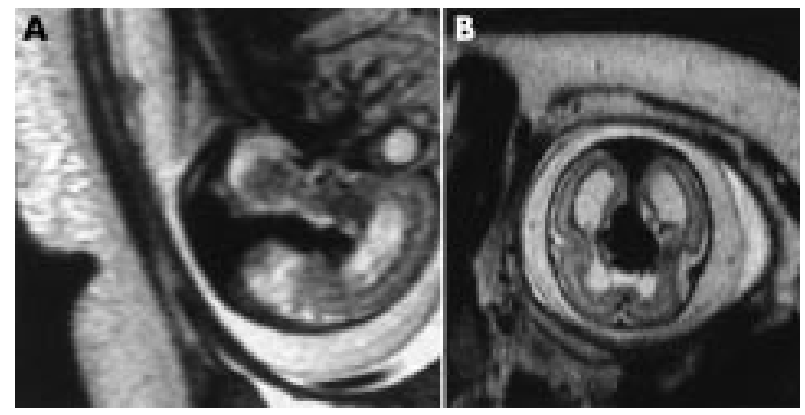

Figure 3 T2 weighted magnetic resonance image. (A) Sagittal and (B) axial view in utero of fetal brain. Antenatal ultrasound detected an abnormality, confirmed on Doppler study as an arteriovenous shunt. The flow void of the VGAM is well shown. Note also the hydrocephalus and oligohydramnios. Fetal cardiac failure can be diagnosed antenatally by pulse rate $>200$ per minute, supraventricular ectopics, and tricuspid insufficiency on ultrasound. Although hydrocephalus may be well tolerated (with open fontanelles), overt cardiac failure has a very poor prognosis. This fetus died in utero.

failure, etc) treatment is performed earlier. Surgery has little role in the modern treatment of VGAM. Surgical attempts at closure of the shunt have a very high mortality or severe morbidity, and shunting of the ventricles before embolisation may accelerate progressive atrophy ("melting brain").

Embolisation in a 5-6 month old baby is technically easier than in a neonate (fig 4). Navigation of microcatheters to the site of the shunt can still be difficult though, because of the often extreme tortuosity of the arterial system. Embolisation is best performed with liquid embolic agents, usually a pure cyanoacrylate glue such as Histoacryl (Braun, Melsungen, Germany) opacified with tantalum powder. We have found newer cyanoacrylates such as Glubran-2 polymerise too slowly for safe use in this high flow situation. The alternative transtorcular venous approach (although technically easier) is probably associated with a higher risk of haemorrhagic complications (and lower success rate) and we have normally avoided this route.

Restraints on the amount of parenteral fluid that can be given to a neonate or infant (contrast medium, flush solutions, and so on) impose limits on the duration of the procedure. Often a large shunt with many feeding vessels will need several embolisation sessions. We have varied from complete occlusion of small lesions in a single session, to a maximum of six sessions for complete occlusion.

Progressive stenosis of the jugular bulbs complicates the course of many VGAM babies. In the neonate, cortical veins do not yet communicate with the cavernous sinus and there is thus no other route for venous outflow. Once cavernous capture, by the cortical venous system, occurs (during the first year) alternative pathways become available (via the ophthalmic and pterygoid veins). Stenting of the jugular bulb in VGAM babies was first described by Dr Wendy Taylor's group at Great Ormond Street Hospital (now Cromwell Hospital) in London. ${ }^{7}$ Although short term results have been promising, the long term patency of stents in the venous system is unknown.

Where treatment is performed before significant brain damage has occurred, a good outcome is anticipated. In Lasjaunias's series, ${ }^{8}$ the largest discussing the management of VGAMs, of 21 babies diagnosed antenatally, 14 were considered fit for embolisation and 12 of these had normal neurological development. In the neonatal diagnosis group of 50 babies, 19 had pre-existing irreversible damage, and 29 were considered fit for embolisation, $50 \%$ of these showing 


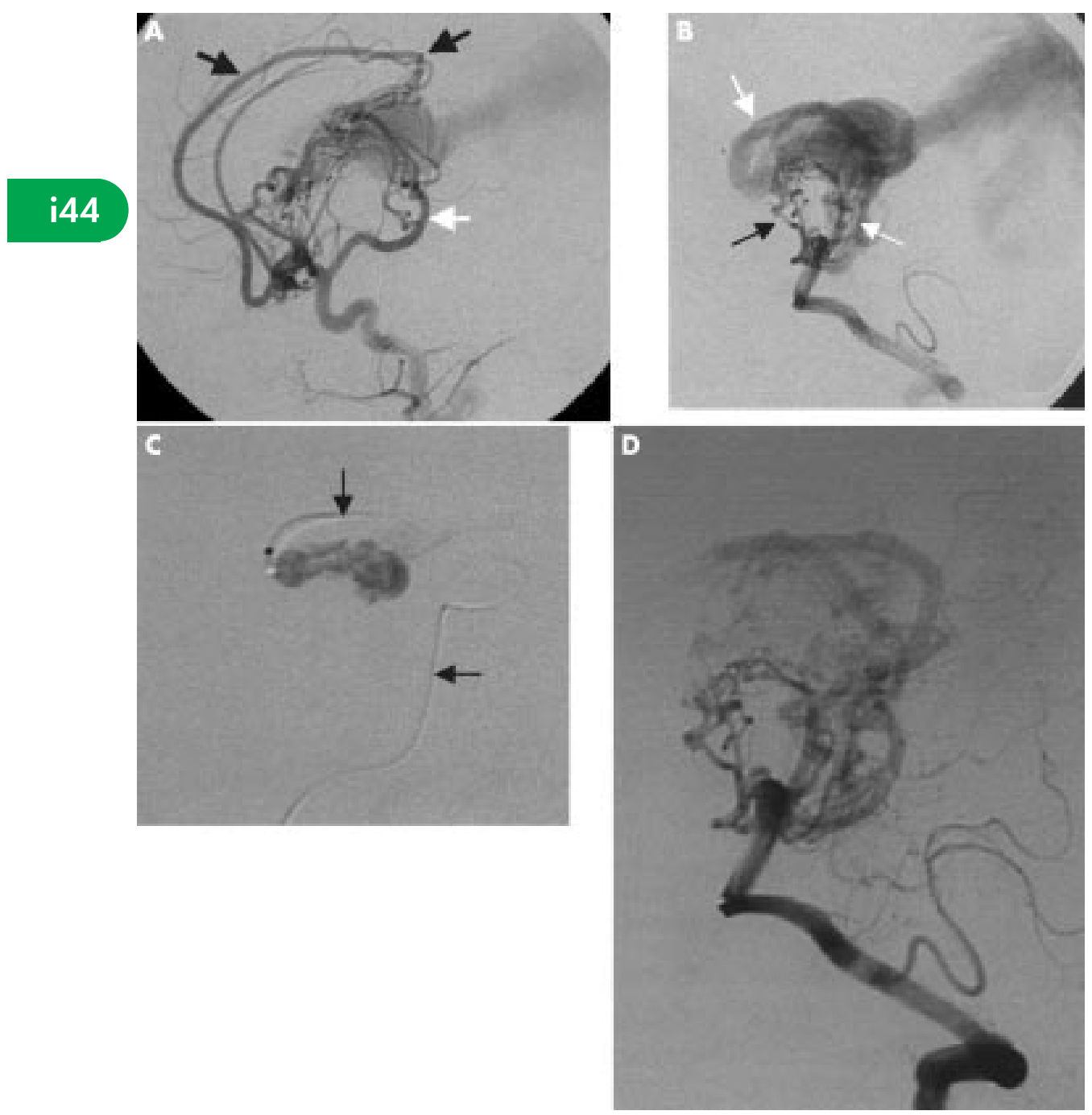

Figure 4 VGAM diagnosed at age 4 days. Mild cardiac failure was treated with diuretics until age 5 months. (A) Right internal carotid artery shows arterial supply to the VGAM from the anterior cerebral artery (limbic arch, black arrows). (B) Left vertebral artery angiogram shows main supply from the posterior choroidal arteries (white arrows), with small subependymal artery contributions (black arrows). (C) Flow guided microcatheter (arrows) has been navigated via posterior choroidal feeders to the shunt. Note the recoil of the catheter tip during rapid glue injection. Stiffer microcatheters show less recoil, but are more difficult to navigate. The cast of cyanoacrylate glue is opacified with tantalum powder. (D) Post-embolisation angiogram shows considerable reduction in shunt. This may progress to complete occlusion. At follow up, if the shunt persisted, further embolisation may be needed.

permanent neurological deficits. In the infant presentation group of 35 babies, embolisation was performed in 26 with 22 having a good neurological outcome. Twelve children presented later, nine requiring embolisation and six of these having a normal course. The worst prognosis was thus seen, as expected, in the babies with the largest shunts, presenting as neonates with severe cardiac failure.

Untreated VGAMs have a poor prognosis and are almost always fatal, but it is equally important to recognise that treatment may be inappropriate in some cases. Where there is evidence of pre-existing brain damage (progressive atrophy or "melting brain syndrome", parenchymal calcification, and so on) or severe multiorgan failure, a poor outcome (death or survival with severe brain damage) is inevitable.

\section{CONCLUSIONS}

High flow arteriovenous shunts of the brain in young children are among the most challenging conditions treated in modern medicine. Early and appropriate diagnostic tests (mainly ultrasound and magnetic resonance imaging) permit the proper management by a team that should include interventional neuroradiologists, neonatologists, paediatric cardiologists, and neurologists. Where possible, VGAMs are treated medically until 5-6 months of age, while unstable babies require early treatment, principally with cyanoacrylate embolisation.

\section{Authors' affiliations}

J J Bhattacharya, Department of Neuroradiology, Institute of Neurological Sciences, Southern General Hospital, Glasgow, UK J Thammaroi, Department of Radiology, Srinagarind Hospital, Khon Kaen University, Thailand

\section{REFERENCES}

1 Thammaroi J, Bhattacharya JJ. Interventional neuroradiology in neonates and children. Radiology Now 2002;19(3):8-11.

2 Gomez DG, Dibenetto AT, Pavese AM, et al. Development of arachnoid villi and granulations in man. Acta Anat (Basel) 1981;11:247-58.

3 Lasjaunias PL. Vascular diseases in neonates infants and children. Heidelberg: Springer, 1997:31-40.

4 Chul Suh D, Alvarez H, Bhattacharya JJ, et al. Intracranial haemorrhage within the first two years of life. Acta Neurochir (Wien) 2001;143:9971004 .

5 Bhattacharya JJ, Luo CB, Suh DC, et al. Wyburn-Mason or Bonnet-Dechaume-Blanc as cerebral arteriovenous metameric syndromes (CAMS): a new concept and a new classification. Interventional Neuroradiology 2001;7:5-17.

6 Raybaud CA, Strother CM, Hald JK. Aneurysms of the vein of Galen: embryonic considerations and anatomical features relating to the pathogenesis of the malformation. Neuroradiology 1989;31:109-28.

7 Brew S, Taylor W, Reddington A. Stenting of a venous stenosis in vein of Galen malformation. Interventional Neuroradiology 2001;7:237-40.

8 Lasjaunias $\mathbf{P}$, Alvarez H, Rodesch $G$, et al. Aneurysmal malformation of the vein of Galen, follow up of 120 children treated between 1984 and 1994. Interventional Neuroradiology 1996;2:15-26. 